DOI https://doi.org/10.24297/jam.v20i.8978

\title{
A Variable Structural Control for a Hybrid Hyperbolic Dynamic System
}

\author{
Xuezhang Hou \\ Mathematics Department, Towson University, Baltimore, Maryland 21252-0001 \\ Email: xhou@towson.edu
}

\begin{abstract}
In this paper, we are concerned with a hybrid hyperbolic dynamic system formulated by partial differential equations with initial and boundary conditions. First, the system is transformed to an abstract evolution system in an appropriate Hilbert space, and spectral analysis and semigroup generation of the system operator is discussed. Subsequently, a variable structural control problem is proposed and investigated, and an equivalent control method is introduced and applied to the system. Finally, a significant result that the state of the system can be approximated by the ideal variable structural mode under control in any accuracy is derived and examined.
\end{abstract}

Keywords: Partial differential equations, Hyperbolic dynamic system, Variable structural control, Semigroup of linear operators.

AMS subject classifications: 93C20, 93D15, 35B35, 35P10

\section{Introduction}

A great attention has been paid to the dynamics and control of flexible beam (see [1]-[8]). In this paper, we are concerned with the following general hyperbolic dynamic system with static boundary condition in one space variable in normal form studied in [1]-[3]:

$$
\left\{\begin{array}{l}
\frac{\partial}{\partial t}\left[\begin{array}{l}
u(x, t) \\
v(x, t)
\end{array}\right]+K(x) \frac{\partial}{\partial x}\left[\begin{array}{l}
u(x, t) \\
v(x, t)
\end{array}\right]+C(x)\left[\begin{array}{c}
u(x, t) \\
v(x, t)
\end{array}\right]=0,0<x<1, t>0, \\
v(1, t)=D u(1, t), u(0, t)=E v(0, t)
\end{array}\right.
$$

where

(H1) $K(x)=\operatorname{diag}\left\{\lambda_{1}(x), \lambda_{2}(x), \cdots, \lambda_{m}(x), \mu_{1}(x), \mu_{2}(x), \cdots, \mu_{k}(x)\right\}$ is a diagonal $n \times n,(n=m+k)$, matrix with real entries $\lambda_{j}(x), \mu_{j}(x) \in C^{1}[0,1], \lambda_{j}(x)>0, \mu_{i}(x)<0, \forall x \in[0,1], i=1,2, \cdots, k, j=1,2, \cdots, m$.

(H2) $C(x)=\operatorname{diag}\left\{c_{1}(x), c_{2}(x), \cdots, c_{n}(x)\right\}$ is an $n \times n$ diagonal matrix with continuous entries in $x \in[0,1]$;

(H3) $u(x)=\left[u_{1}(x), u_{2}(x), \cdots, u_{m}(x)\right]^{\top}$ is a column vector in $\mathbb{R}^{m}\left(\right.$ or $\left.\mathbb{C}^{m}\right)$ and $v(x)=\left[v_{1}(x), v_{2}(x), \cdots, v_{k}(x)\right]^{\top}$ is a column vector in $\mathbb{R}^{k}$ (or $\left.\mathbb{C}^{k}\right)$;

(H4) $D, E, F$ and $G$ are real (or complex) constant matrices of appropriate size.

In order to investigate the variable structural control problem for the system, first, let's transfer the system to an abstract Cauchy problem in an appropriate Hilbert space, then discuss the spectral properties and semigroup generation 
of the system operator.

\section{Spectral Analysis and Semigroup Generation}

We start this section with considering the system (1.1) in the underlying Hilbert space $\mathcal{H}=\left(L^{2}(0,1)\right)^{2}$. Define the operator $\mathcal{A}: D(\mathcal{A})(\subset \mathcal{H}) \rightarrow \mathcal{H}$ by

$$
\left\{\begin{array}{l}
\mathcal{A}\left[\begin{array}{l}
u(x) \\
v(x)
\end{array}\right]=-K(x) \frac{\partial}{\partial x}\left[\begin{array}{l}
u(x) \\
v(x)
\end{array}\right]-C(x)\left[\begin{array}{l}
u(x) \\
v(x)
\end{array}\right], \\
D(\mathcal{A})=\left\{[u, v]^{\top} \in\left(H^{1}(0,1)\right)^{m} \times\left(H^{1}(0,1)\right)^{k}, u(0)=E v(0), v(1)=D u(1)\right\} .
\end{array}\right.
$$

Then the system (2.1) can be written an an evolution equation in $\mathcal{H}$ :

$$
\frac{d W(t)}{d t}=\mathcal{A} W(t), t>0
$$

with $W(t)=[u(\cdot, t), v(\cdot, t)]^{\top}$.

Lemma 2.1 The operator $\mathcal{A}$ defined by (2.2) has compact resolvent and hence $\sigma(\mathcal{A})$ consists only isolated eigenvalues.

Proof. Given $(f, g, b) \in X$, we solve

$$
(\lambda-\mathcal{A})(u, v, d)=(f, g, b)
$$

that is,

$$
\left\{\begin{array}{l}
\frac{\partial}{\partial x}\left[\begin{array}{l}
u(x, t) \\
v(x, t)
\end{array}\right]=-K^{-1}(x)[\lambda+C(x)]\left[\begin{array}{c}
u(x, t) \\
v(x, t)
\end{array}\right]+K^{-1}(x)\left[\begin{array}{c}
f(x) \\
g(x)
\end{array}\right], 0<x<1, t>0 \\
v(1, t)=D u(1, t), u(0, t)=E v(0, t)
\end{array}\right.
$$

Let's denote by $M(x, y, \lambda)$ the fundamental matrix of the system

$$
\frac{d}{d x}\left[\begin{array}{l}
u(x) \\
v(x)
\end{array}\right]=-K^{-1}(x)[\lambda+C(x)]\left[\begin{array}{l}
u(x) \\
v(x)
\end{array}\right]
$$

It follows from (2.3) that

$$
\left[\begin{array}{l}
u(x) \\
v(x)
\end{array}\right]=M(x, 0, \lambda)\left[\begin{array}{c}
E \\
I
\end{array}\right] v(0)+\int_{0}^{x} M(x, y, \lambda) K^{-1}(y)\left[\begin{array}{c}
f(y) \\
g(y)
\end{array}\right] \mathrm{d} y
$$

On the other hand, we see from the boundary condition in (2.1) that

$$
\begin{array}{r}
b=(-\lambda D-F, \lambda-G)\left[\begin{array}{c}
u(1) \\
v(1)
\end{array}\right]=(\lambda D-F, \lambda-G) M(1,0, \lambda)\left[\begin{array}{c}
E \\
I
\end{array}\right] v(0) \\
+(\lambda D-F, \lambda-G) \int_{0}^{1} M(1, y, \lambda) K^{-1}(y)\left[\begin{array}{c}
f(y) \\
g(y)
\end{array}\right] \mathrm{d} y
\end{array}
$$


Subsequently,

$$
H(\lambda) v(0)=b+\int_{0}^{1}(\lambda D+F, G-\lambda) M(1, y, \lambda) K^{-1}(y)\left[\begin{array}{l}
f(y) \\
g(y)
\end{array}\right] \mathrm{d} y
$$

where

$$
H(\lambda)=-(\lambda D+F, G-\lambda) M(1,0, \lambda)\left[\begin{array}{c}
E \\
I
\end{array}\right]
$$

Defining $h(\lambda)=\operatorname{det} H(\lambda)$, we see that $\lambda \in \sigma(\mathcal{A})$ if and only if $\lambda$ is a zero of the entire function $h(\lambda)$. When $h(\lambda) \neq 0$, $\lambda \in \rho(\mathcal{A})$ and $R(\lambda, \mathcal{A})(f, g, b)=(u, v, d)$ where $(u, v)$ is given by $(2.5)$ with $v(0)$ determined by $(2.6)$ and $d=v(1)-D u(1)$. It can be eventually seen from $(2.5)$ that $R(\lambda, \mathcal{A})$ is compact for any $\lambda \in \rho(\mathcal{A})$.

Theorem 2.2. The operator $\mathcal{A}$ defined by (2.1) generates a $C_{0}$-semigroup $T(t)$ on $\mathcal{H}$.

Proof. We need only to prove the assertion for the case $C \equiv 0$ because is a bounded operator by assumption ( $H 2)$, and bounded perturbations do not affect $C_{0}$-semigroup generations. For the sake of simplicity, we assume that $\mathcal{H}$ is real. The idea is to define an equivalent norm on $\mathcal{H}$ by properly choosing some positive weighting functions $f_{i}(x), 1 \leq i \leq N$ and $g_{i}(x), N+1 \leq i \leq n$, namely, define the norm on $\mathcal{H}$ as

$$
\|(u, v, d)\|^{p}=\sum_{i=1}^{N} \int_{0}^{1} f_{i}(x)\left|u_{i}(x)\right|^{p} \mathrm{~d} x+\sum_{j=N+1}^{n} \int_{0}^{1} g_{j}(x)\left|v_{j}(x)\right|^{p} \mathrm{~d} x+\sum_{j=N+1}^{n}\left|d_{j}\right|^{p}
$$

It is easily verified that $\mathcal{H}^{*}$, the dual space of $\mathcal{H}$, consisting of all elements $\left(u^{*}, v^{*}, d^{*}\right)$ with

$$
\begin{aligned}
& u_{i}^{*}(x)=\|\left((u, v, d) \|^{2-p}\left|u_{i}(x)\right|^{\frac{p}{q}} \operatorname{sign}\left(u_{i}(x)\right), \quad 1 \leq i \leq N,\right. \\
& v_{j}^{*}(x)=\|\left((u, v, d) \|^{2-p}\left|v_{j}(x)\right|^{\frac{p}{q}} \operatorname{sign}\left(v_{j}(x)\right), \quad N+1 \leq j \leq n,\right. \\
& d_{j}^{*}(x)=\|\left((u, v, d) \|^{2-p}\left|d_{j}\right|^{\frac{p}{q}} \operatorname{sign}\left(d_{j}\right), \quad N+1 \leq j \leq n .\right.
\end{aligned}
$$

where $q$ denotes the conjugate number of $\mathrm{p}$, which satisfies $\frac{1}{p}+\frac{1}{q}=1$.

For any $(u, v, d) \in D(A), \quad(u, v, d) \neq 0$ and any $\left(u^{*}, v^{*}, d^{*}\right) \in F((u, v, d)) \subset \mathcal{H}$, where $F$ denotes the duality set. A direct calculation shows that

$$
\begin{aligned}
& \|(u, v, d)\|^{p-2}\left\langle\left(u^{*}, v^{*}, d^{*}\right), A(u, v, d)\right\rangle \\
= & \sum_{i=1}^{N} \int_{0}^{1}-\lambda_{i}(x) f_{i}(x) \frac{\mathrm{d}}{\mathrm{d} x}\left|u_{i}(x)\right|^{p} \mathrm{~d} x+\sum_{j=N+1}^{n} \int_{0}^{1}-\mu_{j}(x) g_{j}(x) \frac{\mathrm{d}}{\mathrm{d} x}\left|v_{j}(x)\right|^{p} \mathrm{~d} x \\
& +\left\langle F u(1)+G v(1),[v(1)-D u(1)]^{\prime \prime}\right\rangle \\
= & -\sum_{i=1}^{N} \lambda_{i}(1) f_{i}(1)\left|u_{i}(1)\right|^{p}-\sum_{j=N+1}^{n} \mu_{j}(1) g_{j}(1)\left|v_{j}(1)\right|^{p} \\
& +\sum_{i=1}^{N} \lambda_{i}(0) f_{i}(0)\left|u_{i}(0)\right|^{p}+\sum_{j=N+1}^{n} \mu_{j}(0) g_{j}(0)\left|v_{j}(0)\right|^{p} \\
& +\sum_{i=1}^{N} \int_{0}^{1}\left|u_{i}(x)\right|^{p} \frac{\mathrm{d}}{\mathrm{d} x}\left[\lambda_{i}(x) f_{i}(x)\right] \mathrm{d} x+\sum_{j=N+1}^{n} \int_{0}^{1}\left|v_{j}(x)\right|^{p} \frac{\mathrm{d}}{\mathrm{d} x}\left[\mu_{j}(x) g_{j}(x)\right] \mathrm{d} x \\
& +\left\langle F u(1)+G v(1),[v(1)-D u(1)]^{\prime \prime}\right\rangle=I_{1}+I_{2}+I_{3}+I_{4} \cdot
\end{aligned}
$$

We estimate $I_{i}$ separately. It is clear from the expression of $I_{3}$ that

$$
I_{3} \leq C_{0}\|(u, v, d)\|^{p}
$$


where $\left.C_{0}=\max i, j \max _{x \in[0,1]}\left\{\frac{\mathrm{d}}{\mathrm{d} x}\left[\lambda_{i}(x) f_{i}(x)\right], \frac{\mathrm{d}}{\mathrm{d} x}\left[\mu_{j}(x) g_{j}(x)\right] \mathrm{d} x\right]\right\}$. Nothing that $u_{i}(0)=\sum_{j=N+1}^{n} e_{i j} v_{j}(0)$, we see that

$$
\begin{aligned}
I_{2} & =\sum_{i=1}^{N} \lambda_{i}(0) f_{i}(0)\left|u_{i}(0)\right|^{p}+\sum_{j=N+1}^{n} \mu_{j}(0) g_{j}(0)\left|v_{j}(0)\right|^{p} \\
& \leq \sum_{j=N+1}^{n}\left[\mu_{j}(0) g_{j}(0)+\sum_{i=1}^{N} \lambda_{i}(0) f_{i}(0)\left(\sum_{k=N+1}^{n}\left|e_{i k}\right|^{q}\right)^{\frac{p}{q}}\right]\left|v_{j}(0)\right|^{p} .
\end{aligned}
$$

Because $\lambda_{i}(0)>0$ and $\mu_{j}(0)<0$ from $(\mathrm{H} 1)$, we can always find $g_{j}(0)>0$ and $f_{i}(0)>0$ such that

$$
\left.\mu_{j}(0) g_{j}(0)+\sum_{i=1}^{N} \lambda_{i}(0) f_{i}(0)\left(\sum_{k=N+1}^{n}\left|e_{i k}\right|^{q}\right)^{\frac{p}{q}}\right] \leq 0, \quad N+1 \leq j \leq n
$$

holds, which implies that $I_{2} \leq 0$.

We now estimate $I_{4}$ by means of the inequalities $(|a|+|b|)^{p} \leq 2^{p}\left(|a|^{p}+|b|^{p}\right)$ and $|a|^{\frac{1}{p}}|b|^{\frac{1}{q}} \leq \frac{|a|}{p}+\frac{|b|}{q}$ which hold for any real $a$ and $b$, we have

$$
\begin{aligned}
I_{4} & \leq \sum_{j=N+1}^{n}\left|\sum_{i=1}^{N} f_{j i} u_{i}(1)+\sum_{i=N+1}^{n} g_{j i} v_{i}(1) \| v_{j}(1)-\sum_{i=1}^{N} d_{j i} u_{i}(1)\right|^{\frac{p}{q}} \\
& \leq \frac{1}{p} \sum_{j=N+1}^{n}\left|\sum_{i=1}^{N} f_{j i} u_{i}+\sum_{i=N+1}^{n} g_{j i} v_{i}(1)\right|^{p}+\frac{1}{q} \sum_{j=N+1}^{n}\left|v_{j}(1)-\sum_{i=1}^{N} d_{j i} u_{i}(1)\right|^{p} \\
& \leq \frac{2^{p}}{p} \sum_{j=N+1}^{n}\left[\left|\sum_{i=1}^{N} f_{j i} u_{i}(1)\right|^{p}+\left|\sum_{i=N+1}^{n} g_{j i} v_{i}(1)\right|^{p}\right]+\frac{1}{q}\|(u, v, d)\|^{p} \\
& \leq \frac{2^{p}}{p} \sum_{j=N+1}^{n}\left(\sum_{i=1}^{N}\left|f_{j i}\right|^{p}\right)^{\frac{p}{q}} \sum_{i=1}^{N}\left|u_{i}(1)\right|^{p}+\frac{2^{p}}{p} \sum_{j=N+1}^{n}\left(\sum_{i=1}^{N}\left|g_{j i}\right|^{p}\right)^{\frac{p}{q}} \sum_{i=N+1}^{n}\left|v_{i}(1)\right|^{p}+\frac{1}{q}\|(u, v, d)\|^{p} \\
& =\sum_{i=1}^{N} \alpha_{i}\left|u_{i}(1)\right|^{p}+\sum_{j=N+1}^{n} \beta_{j}\left|v_{j}(1)\right|^{p}+\frac{1}{q}\|(u, v, d)\|^{p}
\end{aligned}
$$

with $\alpha_{i}$ and $\beta_{j}$ denoting the obvious constants. Subsequently, it can be seen that

$$
\begin{aligned}
& I_{1}+I_{4}-\frac{1}{q}\|(u, v, d)\|^{p} \\
\leq & \sum_{i=1}^{N}\left[-\lambda_{i}(1) f_{i}(1)+\alpha_{i}\right]\left|u_{i}(1)\right|^{p}+\sum_{j=N+1}^{n}\left[\beta_{j}-\mu_{j}(1) g_{j}(1)\right]\left|v_{j}(1)\right|^{p} \\
\leq & \sum_{i=1}^{N}\left[-\lambda_{i}(1) f_{i}(1)+\alpha_{i}\right]\left|u_{i}(1)\right|^{p}+2^{p} \sum_{j=N+1}^{n}\left|\beta_{j}-\mu_{j}(1) g_{j}(1)\right|\left|v_{j}(1)-\sum_{i=1}^{N} d_{j i} u_{i}(1)\right|^{p} \\
& +2^{p} \sum_{j=N+1}^{n}\left|\beta_{j}-\mu_{j}(1) g_{j}(1) \| \sum_{i=1}^{N} d_{j i} u_{i}(1)\right|^{p} \\
\leq & \sum_{i=1}^{N}\left[-\lambda_{i}(1) f_{i}(1)+\alpha_{i}\right]\left|u_{i}(1)\right|^{p}+\sum_{j=N+1}^{n} 2^{p}\left|\beta_{j}-\mu_{j}(1) g_{j}(1) \| v_{j}(1)-\sum_{i=1}^{N} d_{j i} u_{i}(1)\right|^{p}
\end{aligned}
$$




$$
\begin{aligned}
& +2^{p} \sum_{j=N+1}^{n}\left|\beta_{j}-\mu_{j}(1) g_{j}(1)\right|\left(\sum_{i=1}^{N}\left|d_{j i}\right|^{q}\right)^{\frac{p}{q}} \sum_{i=1}^{N}\left|u_{i}(1)\right|^{p} \\
= & \sum_{i=1}^{N}\left[-\lambda_{i}(1) f_{i}(1)+\alpha_{i}+2^{p} \sum_{j=N+1}^{n}\left|\beta_{j}-\mu_{j}(1) g_{j}(1)\right|\left(\sum_{i=1}^{N}\left|d_{j i}\right|^{q}\right)^{\frac{p}{q}}\right]\left|u_{i}(1)\right|^{p} \\
& +\sum_{j=N+1}^{n} 2^{p}\left|\beta_{j}-\mu_{j}(1) g_{j}(1)\right|\left|v_{j}(1)-\sum_{i=1}^{N} d_{j i} u_{i}(1)\right|^{p} .
\end{aligned}
$$

If we choose $f_{i}(1)>0, g_{j}(1)>0$ such that

$$
\left\{\begin{array}{l}
-\lambda_{i}(1) f_{i}(1)+\alpha_{i}+2^{p} \sum_{j=N+1}^{n}\left|\beta_{j}-\mu_{j}(1) g_{j}(1)\right|\left(\sum_{i=1}^{N}\left|d_{j i}\right|^{q}\right)^{\frac{p}{q}} \leq 0 \\
2^{p}\left|\beta_{j}-\mu_{j}(1) g_{j}(1)\right| \leq C
\end{array}\right.
$$

for any $1 \leq i \leq N$ and $N+1 \leq j \leq n$, then

$$
I_{1}+I_{4} \leq\left(C+\frac{1}{q}\right)\|(u, v, d)\|^{p} .
$$

The estimations of $I_{i}$ above show that there exists a constant $M$ such that

$$
\left\langle\left(u^{*}, v^{*}, d^{*}\right), A(u, v, d)\right\rangle \leq M\|(u, v, d)\|^{2}
$$

Now we choose a weighting functions $f_{i}(x)$ and $g_{i}(x)$ such that they satisfy (2.10) and (2.11), and hence define a norm in $\mathcal{H}$ according to (2.3). Because $\mathcal{A}-M$ is dissipative and $\mathcal{A}$ has the properties stated in the Lemma 2.1, we can assert from [9] and [11] that $\mathcal{A}$ generates a $C_{0}$-semigroup on $\mathcal{H}$, and the Theorem 2.2 is established now.

\section{A Variable Structural Control}

Let's establish and discuss a structural control problem for the hybrid hyperbolic system $(2.2)$

$$
\left\{\begin{array}{l}
\frac{d W(t)}{d t}=\mathcal{A} W(t)+B u(W(t), t) \\
W(0)=W_{0}
\end{array}\right.
$$

where $B$ is a bounded linear operator from $\mathcal{H}$ to $\mathcal{H}, u(W, t)$ is the control of the beam system (2.2) that is not continuous on the manifold $S=C W=0$, and $C$ is a bounded linear operator with $S=S(W)=C W \in R^{n}$.

Now, let's consider the $\delta$-neighborhood of sliding mode $S=C W=0$, where $\delta>0$ is an arbitrary given positive number. Using a continuous control $\tilde{u}(W, t)$ to replace $u(W, t)$ in the system (3.1) yields

$$
\left\{\begin{array}{l}
\dot{W}=\mathcal{A} W+B \tilde{u}(W, t) \\
W(0)=W_{0}
\end{array}\right.
$$

where $\dot{W}=\partial W / \partial t$, and the solution of (3.2) belongs to the boundary layer $\|S(W)\| \leq \delta$ 
Let $\dot{S}(W)=C \dot{W}=0$. Applying $C$ to the first equation of (3.2) leads to the following the equivalent control:

$$
u_{e q}(W, t)=-(C B)^{-1} C(\mathcal{A} W)
$$

with assumption that $(C B)^{-1}$ exists. Substitute $u_{e q}(W, t)$ into $(3.1)$ to find

$$
\dot{W}=\left[I-B(C B)^{-1} C\right] \mathcal{A} W
$$

Denote $P=B(C B)^{-1} C$ and $\mathcal{A}_{1}=(I-P) \mathcal{A}$, then $(3.1)$ becomes

$$
\dot{W}=\mathcal{A}_{1} W, \quad W(0)=W_{0}
$$

In the rest part of this paper, we are going to show that the actual sliding mode $W(t)$ will approach uniformly to the ideal sliding mode $\bar{W}(t)$ under certain conditions.

Lemma 3.1 If $(C B)^{-1}$ is a compact operator and $P \mathcal{A}=\mathcal{A} P$, then $\mathcal{A}_{1}=(I-P) \mathcal{A}$ generates a $C_{0}$-semigroup $T_{2}(t)$ in $\mathcal{H}$ and $T_{2}(t)=(I-P) T_{1}(t)$, where $T_{1}(t)$ is the $C_{0}$-semigroup generated by $\mathcal{A}$.

Proof: Inasmuch as $(C B)^{-1}$ is a compact operator, $B$ and $C$ are bounded linear operators, we see from the definition of $P$ that $P$ is compact, and therefor the range of $I-P$ is a closed subspace of $\mathcal{H}$. Since $P^{2}=P$ and $(1-P)^{2}=I-P, I-P$ can be viewed as the identity operator on $(I-P) \mathcal{H}$. It can be easily seen that $T_{2}(t)=(I-P) T_{1}(t)$ is a $C_{0}$-semigroup in $(I-P) \mathcal{H}$.

Next, we shall prove that the infinitesimal generator of $T_{2}(t)$ is $(I-P) \mathcal{A}$ and $\mathcal{D}((I-P) \mathcal{A})=(I-P) \mathcal{D}(\mathcal{A})$.

In fact, for every $x \in(I-P) \mathcal{D}(\mathcal{A})$, there is a $x_{1} \in \mathcal{D}(\mathcal{A})$ such that $x=(I-P) x_{1}$. It should be noted that $T_{1}(t)$ and $I-P$ are commutative because $\mathcal{A}$ and $P$ are commutative. We see that

$$
\begin{gathered}
\lim _{t \rightarrow 0^{+}} T_{2}(t) x-x=\lim _{t \rightarrow 0^{+}}(I-P) T_{1}(t)(I-P) x_{1}-(I-P) x_{1} \\
=\lim _{t \rightarrow 0^{+}}(I-P)^{2} T_{1}(t) x_{1}-(I-P) x_{1} \\
=\lim _{t \rightarrow 0^{+}}(I-P) T_{1}(t) x_{1}-(I-P) x_{1} \\
=(I-P) \lim _{t \rightarrow 0^{+}} T_{1}(t) x_{1}-x_{1} \\
=(I-P) \mathcal{A} x_{1} .
\end{gathered}
$$

Let $\tilde{\mathcal{A}}$ denote the infinitesimal generator of $T_{2}(t)$. Since the limit on the left exists, we can assert that $x \in \mathcal{D}(\tilde{\mathcal{A}})$ and $(I-P) \mathcal{D}(\mathcal{A}) \subseteq \mathcal{D}(\tilde{\mathcal{A}})$.

On the other hand, for any $x \in \mathcal{D}(\tilde{\mathcal{A}})$, since $\mathcal{D}(\tilde{\mathcal{A}}) \subseteq(I-P) \mathcal{H}$, there exists $\tilde{x} \in \mathcal{H}$, such that $x=(I-P) \tilde{x}$, and

$$
\begin{gathered}
\lim _{t \rightarrow 0^{+}} T_{2}(t) x-x=\lim _{t \rightarrow 0^{+}} T_{2}(t)(I-P) \tilde{x}-(I-P) \tilde{x} \\
=\lim _{t \rightarrow 0^{+}}(I-P) T_{1}(t) \tilde{x}-(I-P) \tilde{x} \\
=(I-P) \lim _{t \rightarrow 0^{+}} T_{1}(t) \tilde{x}-\tilde{x} \\
=(I-P) \mathcal{A} \tilde{x}
\end{gathered}
$$


Since the limit of the left hand side exists, and so the limit of the right hand side exists, and $\tilde{x} \in \mathcal{D}(\mathcal{A})$ which implies that $\mathcal{D}(\tilde{\mathcal{A}}) \subseteq(I-P) \mathcal{D}(\mathcal{A})$. Thus, $\mathcal{D}(\tilde{\mathcal{A}})=(I-P) \mathcal{D}(\mathcal{A})$ and $\tilde{\mathcal{A}}$, the infinitesimal generator of $T_{2}(t)$, is $(I-P) \mathcal{A}$. The proof of the lemma is complete.

Theorem 3.2 Suppose that in the hyperbolic system (3.1),

1. $(C B)^{-1}$ exists and it is compact,

2. $P \mathcal{A}=\mathcal{A} P$, where $P=B(C B)^{-1} C$.

Then for any solution $W(t)$ of the system (3.4) satisfying $S\left(\bar{W}_{0}\right)=0, \bar{W}_{0} \in \mathcal{D}\left(\mathcal{A}_{1}\right)$ and $\left\|W_{0}-\bar{W}_{0}\right\| \leq \delta, W_{0} \in \mathcal{D}(\mathcal{A})$, we have

$$
\lim _{\delta \rightarrow 0}\|W(t)-\bar{W}(t)\|=0
$$

uniformly on $[0, T]$ for any positive number $T$.

Proof: It should be noted from the Theorem 2.3 and Lemma 3.1 that $\mathcal{A}$ and $\mathcal{A}_{0}=(I-P) \mathcal{A}$ are infinitesimal generators of $C_{0}$-semigroups $T_{1}(t)$ and $T_{2}(t)$ respectively. By virtue of theory of semigroup of linear operators, we see that there are positive constants $M_{1}, M_{2}, \omega_{1}$ and $\omega_{2}$ such that

$$
\left\|T_{1}(t)\right\| \leq M_{1} e^{\omega_{1} t}, \quad\left\|T_{2}(t)\right\| \leq M_{2} e^{\omega_{2} t} . \quad(0 \leq t \leq T)
$$

In the boundary layer $\left\|T_{1}(t)\right\| \leq \delta$, let's introduce the equivalent control as follows

$$
u_{e q}(W, t)=-(C B)^{-1} C \mathcal{A} W+(C B)^{-1} C \dot{W}
$$

Substitute (3.6) into $(3,1)$ to find

$$
\dot{W}=(I-P) \mathcal{A} W+P \dot{W}
$$

Hence, the solution of (3.7) can be expressed as follows:

$$
W(t)=T_{2}(t) W_{0}+\int_{0}^{t} T_{2}(t-s) P \dot{W}(s) d s,
$$

and therefore, the solution of (3.4) can be written as

$$
\bar{W}(t)=T_{2}(t) \bar{W}_{0}
$$

Subtracting (3.9) into (3.8) yields

$$
\begin{aligned}
& W(t)-\bar{W}(t) \\
= & T_{2}(t)\left(W_{0}-\bar{W}_{0}\right)+\int_{0}^{t} T_{2}(t-s) P \dot{W}(s) d s
\end{aligned}
$$

Since $P \mathcal{A}=\mathcal{A} P$, we see that $P T_{1}(t)=P T_{1}(t)$. It should be emphasized that $(I-P) P=0$ and $T_{2}(t)=(I-P) T_{1}(t)$, and consequently,

$$
\begin{aligned}
\int_{0}^{t} T_{2}(t-s) P \dot{W}(s) d s & =\int_{0}^{t}(I-P) T_{1}(t-s) P \dot{W}(s) d s \\
& =\int_{0}^{t} T_{1}(t-s)(I-P) P \dot{W}(s) d s \\
& =0,
\end{aligned}
$$


and so it implies from (3.10) and (3.5) that

$$
\|W(t)-\bar{W}(t)\| \leq\left\|T_{2}(t)\right\|\left\|W_{0}-\bar{W}_{0}\right\| \leq M_{2} e^{\omega_{2} T}\left\|W_{0}-\bar{W}\right\|,
$$

Since $\left\|W_{0}-\bar{W}_{0}\right\| \leq \delta$, we have

$$
\|W(t)-\bar{W}(t)\| \leq M_{2} e^{\omega_{2} T} \delta
$$

Thus,

$$
\lim _{\delta \rightarrow 0}\left\|W(t)-\bar{W}_{0}\right\|=0 .
$$

The proof of the theorem is complete.

We see from the Theorem 3.2 that the solution of the beam system can be approximated by ideal sliding mode in any accuracy.

\section{Conclusion}

In the present paper, a variable structural control problem for a hybrid hyperbolic dynamic system dominated by partial differential equations subject to the boundary shear force feedback is investigated. An evolution equation corresponding to the beam system is established in an appropriate Hilbert space. A spectral analysis and semigroup generation of the system operator for the system are studied. Finally, a variable structural control is proposed, and a significant result that the solution of the system can be approximated by the ideal variable structural model under the control is obtained.

\section{References}

[1] A. F. Neves, D. S. Ribeiro and O. Lopes, On the spectrum of evolution operators generated by hyperbolic systems, J. Funct. Anal., 67 (1986), 320-344. (https://doi.org/10.1016/0022-1236(86)90029-7)

[2] B. Z. Guo and Y. H. Luo, Riesz basis property of a second order hyperbolic system with collocated scalar input/output, IEEE Trans. Automatic Control, 47(2002), 693 -698.(https://doi.org/10.1109/9.995052)

[3] B. Z. Guo, Y. Xie and X. Hou, "On Spectrum of a General Petrowsky Type Equation and Riesz Basis of N-Connected Beam with Linear Feedback at Joints", Journal of Dynamical and Control Systems, Vol.10,No.2 (2004), 187-211. (https://doi.org/10.1038/nm0304-211)

[4] B. Z. Guo, Riesz basis property and exponential stability of controlled Euler-Bernoulli beam equations with variable coefficients, SIAM J. Control and Optim., 40(6)(2002),1905 - 1923. (https://doi.org/10.1137/S0363012900372519)

[5] B. Z. Guo, Riesz basis approach to stabilization of a flexible beam with a tip mass, SIAM J Control Optim, 2001, 39: 1736-1747. (https://doi.org/10.1137/S0363012999354880) 
[6] Xuezhang Hou and Sze-Kai Tsui, Control and stability of a torsional elastic robot arms, Journal of Mathematical Analysis and Applications, 2000, 243: 140-162. (https://doi.org/10.1006/jmaa.1999.6666)

[7] Xuezhang Hou and Sze-Kai Tsui, Analysis and control of a two-link and theree joint elastic robot arms. Applied Mathematics and Computations, 2004, 152: 759-777. (https://doi.org/10.1016/S0096-3003(03)00593-9)

[8] K. S. Liu, Energy decay problems in the design of a point stabilizer for connected string vibrating systems. SIAM J. Control Optim, 26 (1988), 1348-1356. (https://doi.org/10.1137/0326076)

[9] R. F. Curtain and H. J. Zwart, An introduction to infinite-dimensional linear system theory, Springer-Verlag, New York (1995).

[10] T. Kato, Perturbation Theory of Linear Operators, Springer-Verlag, New York, 1966. (https://doi.org/10.1007/9783-662-12678-3)

[11] A. Pazy, Semigroups of linear operators and applic ations to partial differential equations.SpringVerlag,Berlin(1983). 